\title{
Vessel Wall MRI Enhancement in Noninflammatory Cerebral Amyloid Angiopathy
}

\author{
Q. Hao, (D) N.M. Tsankova, DH. Shoirah, (D).P. Kellner, and (D) K. Nael
}

\section{ABSTRACT}

SUMMARY: Cerebral amyloid angiopathy is characterized by deposition of amyloid- $\beta$ fibrils in the walls of small-to-medium-sized blood vessels. In this retrospective review of 5 patients with histologically confirmed noninflammatory cerebral amyloid angiopathy, high-resolution vessel wall MRI showed arterial wall enhancement in 2 patients (40\%). Despite common consensus of equating vessel wall enhancement with inflammation, this report demonstrates that $\beta$-amyloid accumulation alone without inflammation can be associated with arterial wall enhancement in a subset of patients.

ABBREVIATIONS: CAA = cerebral amyloid angiopathy; VWMRI = vessel wall MR imaging

C erebral amyloid angiopathy (CAA) is characterized by deposition of amyloid- $\beta$ fibrils in the vessel walls of leptomeningeal and cortical arteries, arterioles, and, less often, capillaries and veins of the central nervous system. ${ }^{1}$ Histologically, Puchtler alkaline Congo Red stain remains the standard diagnostic method for staining amyloid deposition, which can be seen in the adventitia, tunica media, or all layers of vessel wall.

Along the spectrum of amyloid-deposition-related angiopathies, there are 3 entities: 1) CAA (generally there is an absence of wall inflammation); 2) inflammatory CAA (varying amounts of perivascular inflammation are present, including multinucleated giant cells); and 3) amyloid- $\beta$-related angiitis (in which there is destructive vasculitis). ${ }^{2}$ There is often overlap in the clinical presentations of these entities, which may include focal neurologic deficits, cognitive impairment, or seizure related to hemorrhagic or ischemic changes. The clinical diagnosis of CAA relies on clinical history and imaging evidence of microbleeds or lobar hemorrhage that are not explained by other etiologies. ${ }^{1}$

Received October 17, 2019; accepted after revision January 9, 2020.

From the Departments of Neurology (Q.H.), Pathology (N.M.T.), Neurosurgery (H.S., C.P.K.), and Radiology (K.N.), Icahn School of Medicine at Mount Sinai, New York, New York; and Department of Radiological Sciences (K.N.), David Geffen School of Medicine, University of California at Los Angeles, Los Angeles, California.

Paper previously presented, in part, at: Annual Meeting of American Society of Neuroradiology, May 18-23, 2019; Boston, Massachusetts.

Please address correspondence to Kambiz Nael, MD, David Geffen School of Medicine at UCLA, Department of Radiological Sciences, 757 Westwood Plaza, Suite 1621D, Los Angeles, CA, 90095-7532; e-mail: kambiznael@gmail.com; @kambiznael

Indicates article with supplemental on-line table.

http://dx.doi.org/10.3174/ajnr.A6445
High-resolution vessel wall MR imaging (VWMRI) is an emerging technique that is used to assess various vasculopathies, such as atherosclerotic disease, vasculitis, Moyamoya disease, reversible cerebral vasoconstriction syndrome, and dissection. ${ }^{3}$ While vessel wall enhancement using VWMRI has been reported in the inflammatory variety of amyloid-related angiopathies, ${ }^{2,3}$ very little is known about the VWMRI findings in patients with noninflammatory CAA. We report the VWMRI findings in a patient with histologically confirmed CAA.

\section{Brief Report}

A 75-year-old patient with a history of hypertension, diabetes, progressive memory loss, and behavior changes during 6 months presented with worsening of mental status followed by seizures and status epilepticus. The initial neurologic examination revealed impaired attention and language; however, cranial nerves, motor strength, and gait were unremarkable. The patient had fluctuating mental status during the first 3 days and developed a clinical seizure on the fourth day with subsequent nonconvulsive status epilepticus, which was revealed on video-electroencephalography and required multiple antiepileptic agents. MR imaging showed no acute infarction but a few chronic microhemorrhages (Fig 1A) and some white matter changes. Due to the presence of chronic microhemorrhages, the differential diagnosis of vasculitis or amyloid angiopathy was entertained. Conventional angiography showed normal intracranial arteries. VWMRI (3D T1-weighted fast-spin-echo sequence with variable refocusing flip angle acquisition with inherent black-blood effect [sampling perfection with application-optimized contrasts by 


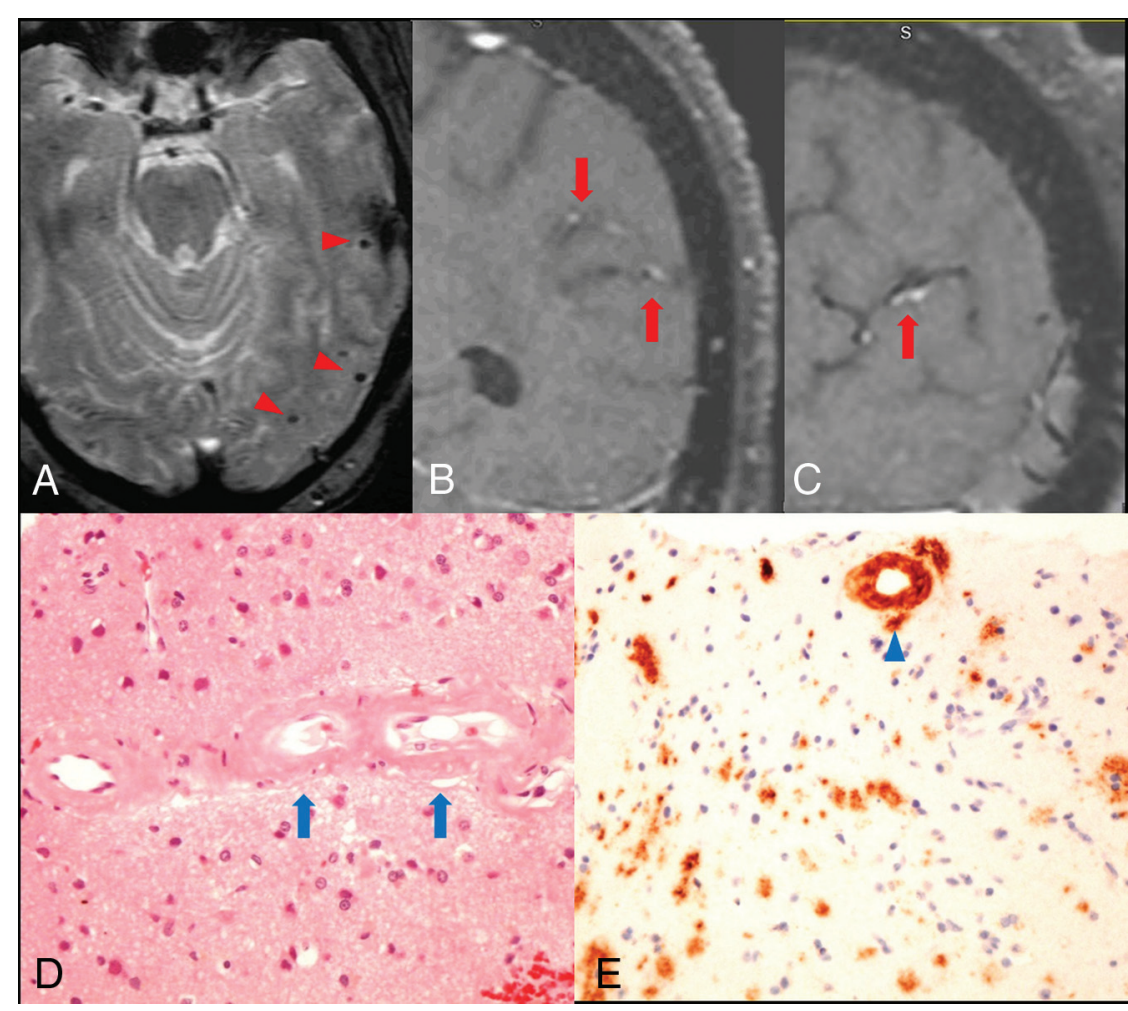

FIG 1. Arterial wall enhancement is associated with amyloid accumulation within the vessel wall. $A$, Gradient-echo image shows microhemorrhages in the left temporal lobe (red arrowheads). $B$ and C, coronal and sagittal views of postcontrast T1-weighted VWMRI show enhancement in the wall of cortical branches of the left middle cerebral artery in the parietal and temporal lobes (red arrows). D, Hematoxylin-eosin stain of a left temporal lobe sample shows thickened, hyalinized blood vessels containing amorphous eosinophilic material (blue arrows) in small- and medium-sized arteries within the leptomeninges and superficial cortical gray matter. $E$, Immunostain for $\beta$-amyloid shows amyloid accumulation within the vessel wall (blue arrowhead). No inflammatory cells were observed surrounding the vessels. rhages, and histopathologic findings are summarized in the On-line Table.

\section{DISCUSSION}

Intracranial vessel wall enhancement detected by high-resolution MR imaging has been considered a sign of inflammation and/or vasa vasorum in-growth in patients with atherosclerosis or vasculitis. ${ }^{3,4}$ Although vessel wall enhancement can be expected with vascular inflammatory processes such as primary angiitis of the central nervous system or an inflammatory type of amyloid such as amyloid $\beta$-related angiitis, in our report, we show that vessel wall enhancement can also be seen in a subset of patients with noninflammatory CAA (40\%). This suggests that $\beta$-amyloid accumulation by itself and without inflammation can also result in vessel wall enhancement. This report, to our knowledge, is the first on VWMRI findings in patients with histopathologically proved noninflammatory CAA. An earlier study by De Havenon et $\mathrm{al}^{5}$ showed vessel wall enhancement in about $50 \%$ of patients with CAA; however, in this study, there was no discrimination between inflammatory and noninflammatory CAA because the patients did not have histopathology to confirm the diagnosis.

One hypothesis or explanation for our findings is potential change and dis-

using different flip angle evolution, SPACE sequence; Siemens, Erlangen, Germany]: FOV, $230 \mathrm{~mm}$; TR/TE, 700/18 ms; matrix, $256 \mathrm{~mm}^{2}$; 144 sections $\times 0.9-\mathrm{mm}$ thick; voxels, $0.9 \times$ $0.9 \times 0.9 \mathrm{~mm}^{3}$ ) on the eighth day of admission showed arterial wall enhancement along the cortical branches of the left middle cerebral artery in the left parietal and temporal lobes (Fig $1 B,-C$ ). Findings of clinical and laboratory work-ups were negative for CNS infection, systemic vasculitis, or metabolic abnormalities except for elevated blood glucose ( $340 \mathrm{mg} / \mathrm{dL}$ on admission). Biopsy revealed $\beta$-amyloid accumulation in the vessel wall without associated inflammation (Fig $1 D,-E)$.

Following institutional review board approval, we subsequently reviewed digital charts of patients with a histologically proved diagnosis of noninflammatory CAA between August 2010 and February 2019. From a total of 10 patients identified, only 4 patients had adequate imaging for inclusion in this study (no MR imaging, $n=2$; inadequate MR imaging image quality to assess vessel wall enhancement, $n=4$ ). Of these 4 patients, 1 demonstrated arterial wall enhancement similar to that in our report. Clinical and demographic data, imaging findings including VWMRI and the number of microhemor- ruption of the blood-brain barrier in CAA. CAA-positive vessels often exhibit morphologic changes such as loss of smooth-muscle cells and vessel wall thickening, which, in turn, can result in increased leakiness and an increased incidence of microbleeds. $^{6,7}$ It is likely that this increased permeability is responsible for wall enhancement in a subset of CAA (despite the absence of inflammation). Most interesting, our patients who exhibited vessel wall enhancement also had numerous microhemorrhages, while those without vessel wall enhancement had none or just a few.

There are several limitations to our study. First, our sample size is very small, precluding any meaningful statistical analysis. Second, there is potential selection bias in this retrospectively collected series. Third, due to the retrospective nature of our study, there may have been variability in our VWMRI sequence parameters. Also, we did not use delay alternating with nutation for tailored excitation (DANTE) preparation to refine our blood suppression. However, we used the best option available to us from our vendors to perform these clinical studies using FDAapproved sequences in a clinical environment. Future prospective radiologic-histologic studies using dedicated VWMRI are needed to better characterize the arterial wall changes in CAA with and 
without inflammation, and their role in CAA-related cerebral pathologies.

Disclosures: Kambiz Nael—UNRELATED: Board Membership: Olea Medical, Comments: Medical Advisory Board. Qing Hao-RELATED: Support for Travel to Meetings for the Study or Other Purposes: Icahn School of Medicine at Mount Sinai, Comments: conference support from departmental research fund.

\section{REFERENCES}

1. Biffi A, Greenberg SM. Cerebral amyloid angiopathy: a systematic review. J Clin Neurol 2011;7:1-9 CrossRef Medline

2. Moussaddy A, Levy A, Strbian D, et al. Inflammatory cerebral amyloid angiopathy, amyloid-beta-related angiitis, and primary angiitis of the central nervous system: similarities and differences. Stroke 2015;46:e210-13 CrossRef Medline
3. Alexander MD, Yuan C, Rutman A, et al. High-resolution intracranial vessel wall imaging: imaging beyond the lumen. $J$ Neurol Neurosurg Psychiatry 2016;87:589-97 CrossRef Medline

4. Lehman VT, Brinjikji W, Kallmes DF, et al. Clinical interpretation of high-resolution vessel wall MRI of intracranial arterial diseases. $\mathrm{Br} \mathrm{J}$ Radiol 2016;89:20160496 CrossRef Medline

5. De Havenon A, Lobo R, Eisenmenger L, et al. MRI detection of vessel wall inflammation and contrast leakage in cerebral amyloid angiopathy. In: Proceedings of the International Stroke Conference, Houston, TX, Feb 22-24, 2017: WP138

6. Freeze WM, Bacskai BJ, Frosch MP, et al. Blood-brain barrier leakage and microvascular lesions in cerebral amyloid angiopathy. Stroke 2019;50:328-35 CrossRef Medline

7. Charidimou A, Boulouis G, Gurol ME, et al. Emerging concepts in sporadic cerebral amyloid angiopathy. Brain 2017;140:1829-50 CrossRef Medline 